\title{
Realtime Logistics: echtzeitnahe Steuerung von Materialflusssystemen auf Basis autonomer Agenten und Entitäten
}

\author{
PROF. DR. MiCHAEL TEN HOMPEL \\ DIPL.-ING. DIRK LIEKENBROCK \\ FRAUNHOFER-INSTITUT FÜR MATERIALFLUSS UND LOGISTIK IML, DORTMUND \\ DiPL.-ING. PETER STUER \\ VANDERLANDE INDUSTRIES LOGISTICS SOFTWARE GMBH, DORTMUND
}

Die Steuerung logistischer und produktionstechnischer Systeme ist heute durchgängig hierarchisch organisiert. Auch dezentrale und wandelbare Systeme mit eingebetteten fraktalen oder adaptiven Elementen oder Regelkreisen lassen sich in ihrer Gesamtheit stets auf eine zeitgenaue zentrale Planung zurückführen. „Realtime Logistics“ bezeichnet im Gegensatz hierzu die echtzeitnahe Materialflusssteuerung auf Basis einer autonomen, selbstgesteuerten Abwicklung der im einzelnen logistischen Objekt implementierten Mission. Hierzu werden mobile Softwareagenten eingesetzt, die zunächst synchron zum Objekt, und in Zukunft eingebettet im logistischen Objekt, laufen werden.

Nowadays, logistic and production systems are controlled consistently hierarchically. Decentralised and changeable systems with embedded fractal or adaptive elements or control cycles are the results of a precisely timed central planning. In contrast to this, "Realtime Logistics" is a realtime material flow control where the mission of the single logistic objects is carried out autonomously and selfcontrolled. Mobile software agents are used for this purpose which at first will operate synchronously to the object and in future will be embedded into the object.

\section{Motivation und Stand der Technik}

In großen Materialflusssystemen wird aufgrund des Zusammenwirkens einer Vielzahl von Einzelelementen die Leistungsfähigkeit des Gesamtsystems nicht allein durch die Leistungsfähigkeit der einzelnen Elemente, sondern insbesondere durch die überlagerten Steuerungsstrategien und -algorithmen bestimmt. Die verwendeten Strategien werden aufgrund der Komplexität der Systeme zumeist über das Arbeitsmittel der Simulation verifiziert. Sehr häufig handelt es sich hierbei um applikationsspezifische Metaheuristiken, die ein hohes Optimierungspotenzial vermuten lassen, das jedoch aufgrund sinkender Inbetriebnahmezeiten nur selten ausgenutzt werden kann.

Zur Abbildung der weiterhin steigenden Dynamik der Auftragslast ist zudem eine kontinuierliche Adaption dieser Strategien elementar. Die resultierenden applikationsspezifischen Lösungen werden im Laufe der Inbetriebnahme und während des Betriebes angepasst, jedoch nur selten in der begleitenden Simulation eingepflegt, was wiederum zur Folge hat, dass der Wert einer betriebsbegleitenden Simulation häufig eher fraglich ist. Dies betrifft explizit die Strategien zur Steuerung der echtzeitnahen (automatisierten) Materialflusstechnik.

Komplexe Materialflusssysteme werden trotz der Fortschritte in der heutigen Automatisierungs- und Steuerungstechnik, von wenigen Prototypen abgesehen, mit hierarchisch organisierten, zunehmend modular aufgebauten speicherprogrammierbaren Steuerungen bedient, die über Jahre hinaus nahezu unverändert in Betrieb bleiben. Die Wandelbarkeit, Flexibilität und Adaptivität dieser Systeme ist durch deren starre Konzeption sehr begrenzt. Änderungen erfordern zumeist die Überarbeitung und Neuinstallation des oft komplexen Steuerungsprogramms, verbunden mit dem vorübergehenden Abschalten der korrespondierenden Fördertechnik.

Ein ökonomischer Betrieb komplexer Materialflusssysteme bedarf aber der regelmäßigen, dynamischen (echtzeitnahen) Modifikation und Adaption der Steuerung, die aufwandsarm und im laufenden Betrieb erfolgen muss.

Am Lehrstuhl für Förder- und Lagerwesen der Universität Dortmund wird unter dem Titel „Realtime Logistics“ zurzeit ein neuartiges, agentenbasiertes Steuerungssystem in Betrieb genommen, welches die beschriebenen systemimmanenten Nachteile durch den Einsatz autonomer Soft- und Hardwarekomponenten zu vermeiden sucht (vgl. Abb. 1). 


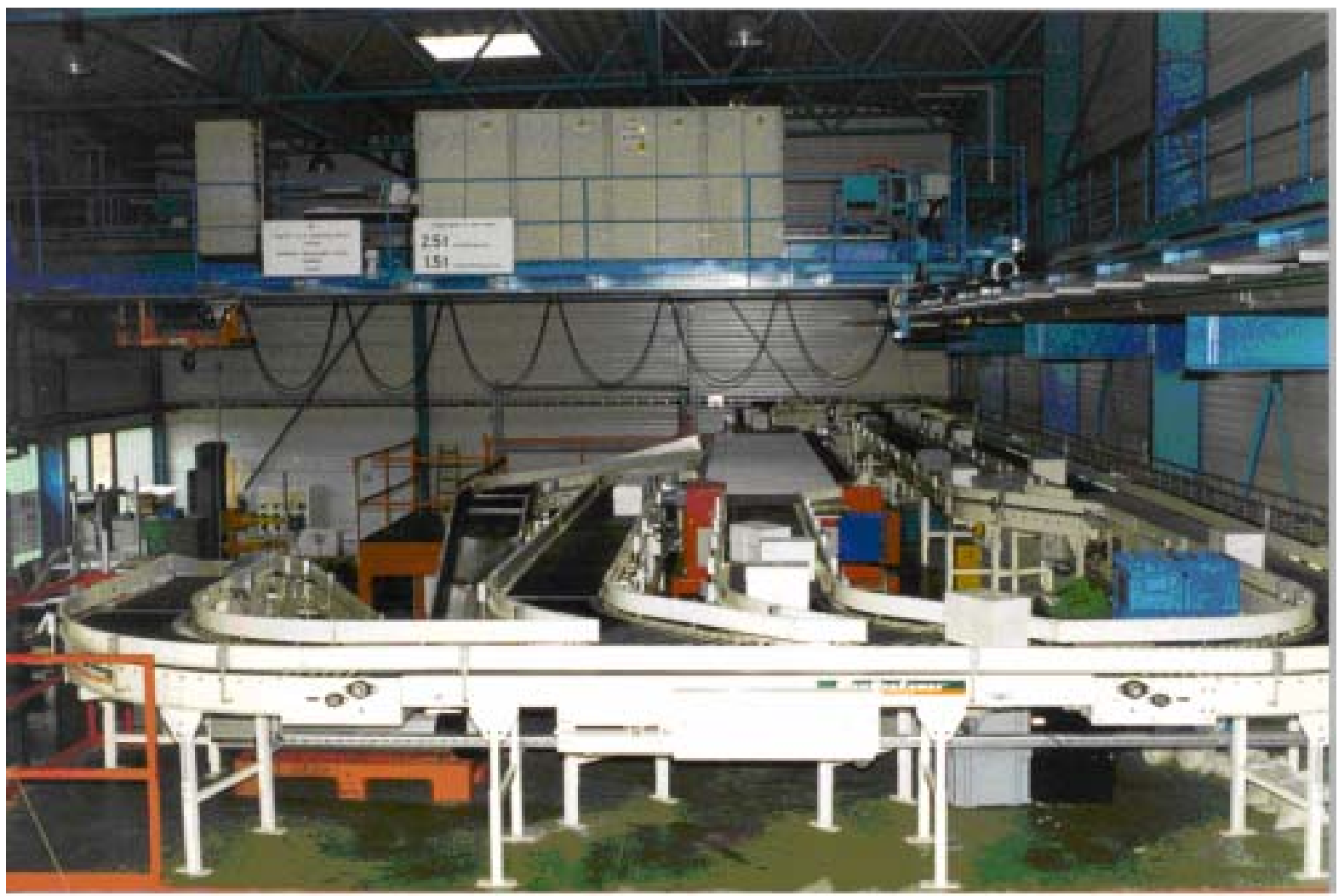

Abbildung 1: Blick auf die obere Ebene der Versuchsanlage

\section{Stand der Dinge auf dem Gebiet der wandelbaren, flexiblen und adaptiven Materialflusssteuerungen}

Heutzutage erfordern dynamische Produktionsstrukturen flexible Arbeitszeitmodelle und hohe Personalkosten, um jederzeit schnell und in hoher Qualität die Wünsche des Kunden erfüllen zu können. Deshalb bedarf es für die Verfolgung der Produktion kundenindividueller Güter in einem turbulenten Auftragsumfeld neuer automatisierter Ansätze in der innerbetrieblichen Logistik, um die Kostenschere gegenüber der Serienfertigung zu verkleinern, wandelbare Materialflusssysteme zu generieren und gleichzeitig eine hohe Verfügbarkeit durch Reduzierung der Komplexität zu gewährleisten [Günthner02].

Seit Mitte der 90er Jahre ist eine wachsende Tendenz zur Dezentralisierung und Standardisierung von Automatisierungskomponenten zu verzeichnen [Meinberg96]; [Günthner98]. Der Einsatz baulich ähnlicher Module mit breitem Anwendungsbereich dient dem Ziel, die Komplexität der Gesamtsysteme durch den Einsatz wiederkehrender Steuerungsmodule zu reduzieren. Die Zusammenführung von Motoren, Frequenzumrichtern, Mikrocontrollern und Kommunikationsmodulen zu kompakten und schnittstellenarmen Systemen ist ein typisches Beispiel hierfür.

Im Zuge dieser Entwicklung werden mittlerweile verschiedene dezentrale Steuerungssysteme unterschiedlicher Komplexität am Markt angeboten und versprechen aufgrund ihrer Modularität und Vernetzbarkeit eine Reihe betrieblicher Vorteile, u.a.:

- $\quad$ erhöhte Verfügbarkeit durch verteilte Hardware

- $\quad$ transparentere, da lokal begrenzte Steuerungsstrukturen

- $\quad$ vereinfachte Vernetzbarkeit durch standardisierte Schnittstellen (Ethernet)

- $\quad$ verbesserte Wartbarkeit/Fernwartung durch standardisierte Schnittstellen

- $\quad$ vereinfachte Ablauflogiken innerhalb einzelner Module

Hervorzuheben ist auch die Vernetzung der Module durch Ethernet und die Verwendung des nicht echtzeitfähigen TCP/IP Protokolls, welches aufgrund seiner großen Verbreitung die Konnektivität und Fernwartung der Module ohne hohen technischen Aufwand ermöglicht. 
Trotz der beschriebenen Vorteile und prinzipbedingten Potenziale werden zwar dezentrale Steuerungssysteme auf Feldbusbasis eingesetzt, die inne wohnende Steuerungslogik ist jedoch zumeist zentral und hierarchisch organisiert. Die „Intelligenz“ vor Ort wird damit nur in Grenzen genutzt und eine stringente logische Modularisierung ist bis dato auf wenige Anwendungen im Produktionsumfeld beschränkt geblieben.

„... Die Wandelbarkeit [von Materialflusssystemen] bedingt in der Umsetzung neben dem Einsatz flexibler Fördersysteme insbesondere auch Automatisierungsstrukturen, die aufwandsarm zu projektieren bzw. zu integrieren sind und damit eine autoadaptive Funktionalität aufweisen, die diese Forderungen erfüllt. Ein weiterführendes Szenario dieser Zielsetzung stellt schließlich die vollständig autonome Steuerung logistischer Prozesse dar...“ [Scholz04]

\section{Realtime Logistics}

Ein wesentliches Kriterium bei der Entwicklung von Steuerungssystemen allgemein ist die Abbildung echtzeitnaher Funktionalität. Diese unabdingbare Voraussetzung beschreibt die Fähigkeit, in einer vorgegebenen Umgebung innerhalb vorbestimmter Zeit auf ein äußeres Ereignis reagieren zu können. Diese (Echtzeit-) Fähigkeit (Realtime Capability) ist in hohem Maße kontextsensitiv und nicht nur von der physischen sondern auch von der logischen Umgebung abhängig. Dies erfordert die autonome Kommunikation sowohl mit anderen logistischen oder materialflusstechnischen Objekten innerhalb der aktuellen Umgebung als auch mit der Umgebung selbst (z. B. in Form der Ambient Intelligence) [tenHompel04].

Diese Fähigkeit in Echtzeit oder echtzeitnah zu kommunizieren und zu reagieren - als Voraussetzung für die Steuerung von Materialflusssystemen auf Basis modularer bzw. autonomer Entitäten ${ }^{1}$ - gab dem Projekt „Realtime Logistics“ den Namen.

Um eine durchgängige Gestaltung und Kommunikation über die Grenzen des traditionellen, SPS-basierten Entwurfes hinaus zu gewährleisten, sind hierbei die Entitäten als Web-Server auf Linux-Basis ausgeführt. Die Kommunikation erfolgt über XML-Standard, der auf allen Ebenen bis hin zur SAP-Integration Anwendung findet. Einerseits wird hierdurch der Transparenz der Kommunikation und der zunehmenden Verbreitung XMLbasierter Web Services als de-facto-Standard informationslogistischer Kommunikation Rechnung getragen, andererseits ist die Einführung des EPC-konformen XML-Dialektes PML zusätzliche Motivation. Die vergleichsweise aufwendige Syntaktik von XML kontakariert zwar den Wunsch nach möglichst leistungsfähiger Kommunikation, in der Realität überschreiten die Latenzzeiten der Datenübertragung die effektiven Kommunikationszeiten jedoch ohnehin erheblich. Die verfügbaren Bandbreiten von $100 \mathrm{MHz}$ und mehr liegen weit oberhalb der Anforderungen. Dies gilt auch für den erhöhten Kommunikationsbedarf, der durch das XMLFormat und die Kommunikation der Entitäten untereinander entsteht.

Die Linux-basierten Entitäten sind jeweils einer definierten Anzahl von Aktoren und Sensoren zugeordnet und steuern die Funktionalität vor Ort (zum Beispiel einer Weiche oder einer Pufferstrecke etc.). Die eigentliche (strategische) Steuerung des materialflusstechnischen Systems erfolgt über autonome, mobile Softwareagenten, die sich zwischen den Entitäten bewegen und als Java-Programm auf den jeweiligen Linux-Web-Servern ablaufen. Die Kommunikation der Agenten und ihre Bewegung erfolgt über den gleichen Kanal (TCP/IP auf Ethernet) wie die Kommunikation der Entitäten selbst.

Jeder Agent wird einer fördertechnischen Einheit (einem Behälter, Paket o.ä.) zugeordnet und mit dem Eintritt in das System (bei erster Identifikation) gestartet. Anschließend bewegt sich der Agent parallel zur fördertechnischen Einheit von Entität zu Entität über das System.

Neben der systemimmanenten Synchronisation von Material- und Informationsfluss liegt in der Kommunikation der mobilen Softwareagenten untereinander und mit ihrer Umwelt (Fördertechnik, Identsysteme etc.) die Möglichkeit zur strategischen Auftragsbearbeitung für jede Entität und damit für das gesamte materialflusstechnische System (vgl. Emergenz). In der Konsequenz werden lediglich eine Auftragsliste und die Topologie des Systems zentral gespeichert, die Auftragsbearbeitung erfolgt dezentral. Die resultierende Leistung des Gesamtsystems wird im Gegensatz zu einer vollständigen Batchvorplanung theoretisch nicht optimal sein, je nach Zielfunktion der Agenten kann jedoch eine sehr hohe Dynamik abgebildet werden.

Die Identifikation und die Speicherung der Quelle/Ziel-Informationen erfolgt über RFID-Tags am Fördergut. Nahe liegender Weise kann zukünftig - entsprechend leistungsfähige und preiswerte Chip-Technologie vorausgesetzt - der Agent alternativ auf dem Tag gespeichert werden (Abb. 2).

\footnotetext{
${ }^{1}$ Als Entität wird in diesem Zusammenhang die physische Repräsentanz im Sinne eines „Steuerungsknoten“ oder „steuerungstechnischen Moduls“ verstanden. Die allgemeine Bezeichnung Entität wurde gewählt, um Verwechselungen mit den Elementen klassischen dezentraler oder modularer Steuerungssysteme zu vermeiden. Entitäten besitzen in diesem Zusammenhang immer Server-Funktionalität.
} 
DOI: 10.2195/LJ_Not_Ref_d_tenHompel_0320052
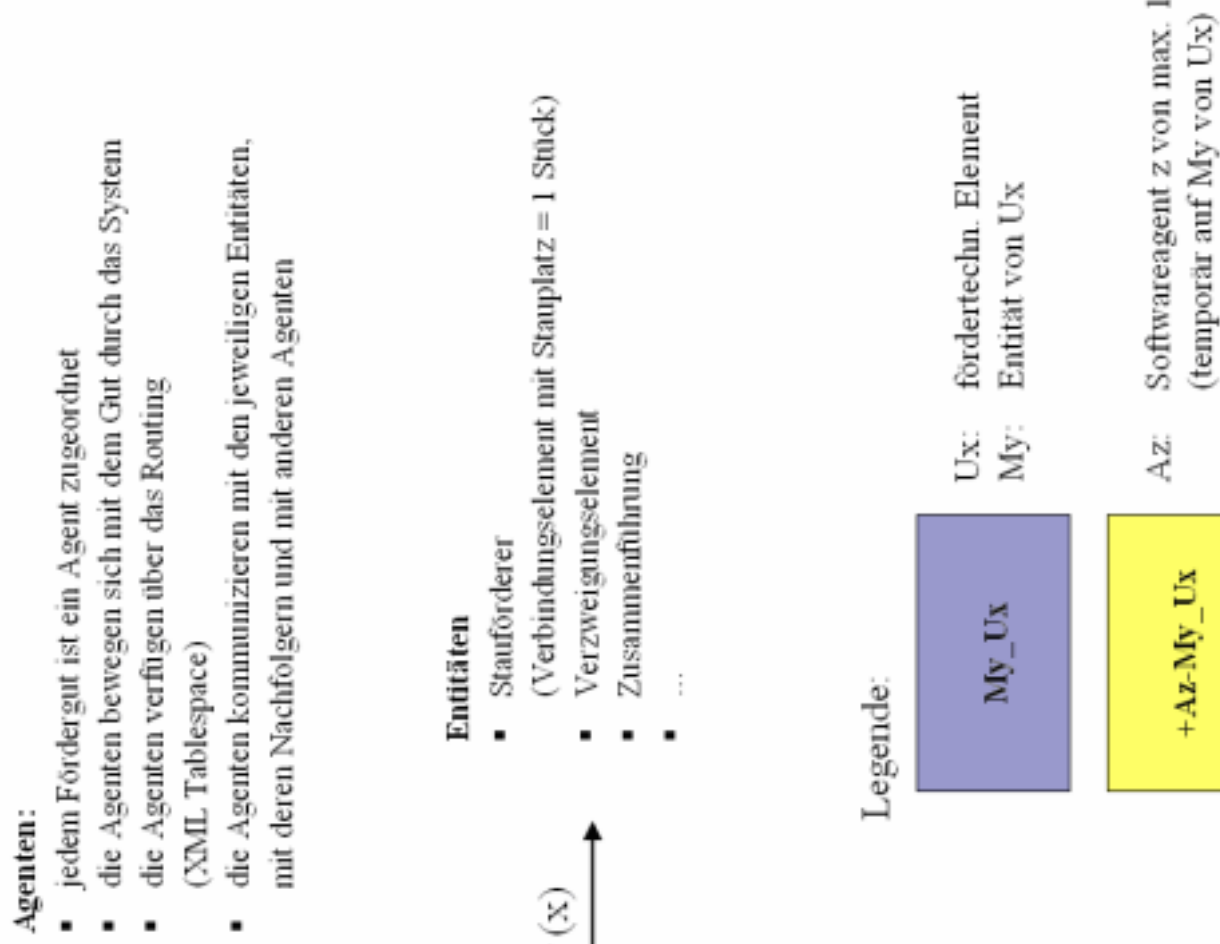

$\stackrel{2}{2}$

บุi
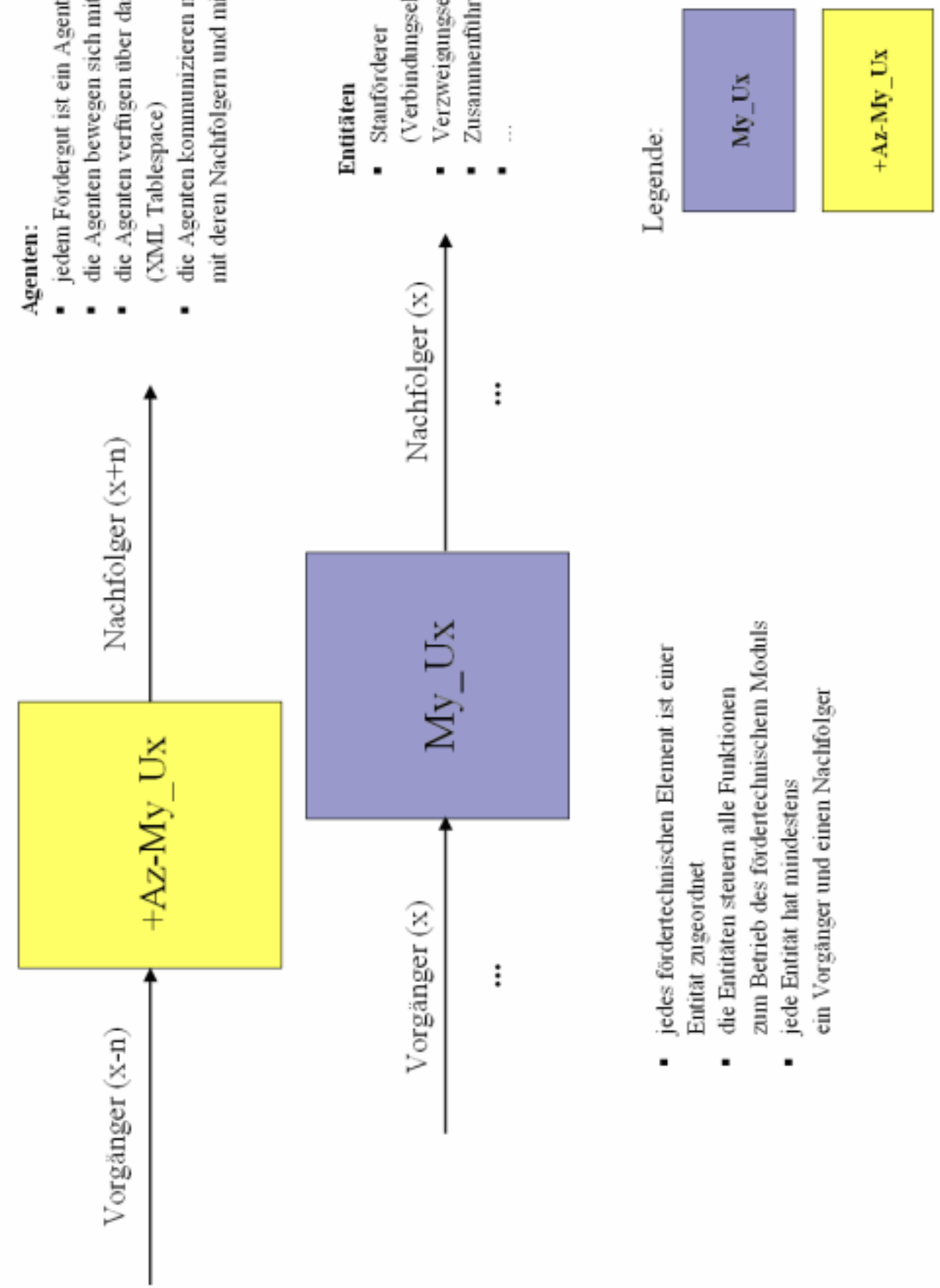

Abbildung 2: Agenten und Entitäten 


\section{Literatur}

[Günthner98]

[Günthner02]

[Günthner03]

[Krum2001]

[Meinberg96]

[Scholz04]

[tenHompel04]
Günthner, Willibald: Dynamik im Griff. In: Logistik Heute (1998) 12 Günthner, Willibald: Anforderungen an automatisierte Materialflusssysteme für wandelbare Logistikstrukturen. In: Tagungsband Wissenschaftssymposium Logistik der BVL 2002, Huss-Verlag

Günthner, Willibald: Materialflusstechnologie - Anforderungen und Konzepte für wandelbare Materialflusssysteme, 21. Dortmunder Gespräche, September 2003 Krumnacker, Christoph: Konzeption eines agentenbasierten Modells für flexible Informationsschnittstellen in komplexen Logistiksystemen, Dissertation, Praxiswissen Verlag, 2001

Meinberg, Uwe: Chancen mit dezentralen Steuerungen. In: Frankfurter Allgemeine Zeitung, Ausgabe vom 22.08.96

Scholz-Reiter, Bernd; Freitag, Michael; Herzog, Otthein: Selbststeuerung logistischer Prozesse - Ein Paradigmenwechsel und seine Grenzen. Industrie Management 20 (2004) 1, GITO-Verlag

ten Hompel, Michael; Stuer, Peter: Realtime logistics. In: Fördertechnik Jahressonderheft, 2004 